\title{
Multiple Scattering Tomography
}

\author{
Peter Modregger, ${ }^{1,2, *}$ Matias Kagias, ${ }^{1,3}$ Silvia Peter, ${ }^{1,3}$ Matteo Abis, ${ }^{1,3}$ Vitaliy A. Guzenko, ${ }^{4}$ \\ Christian David, ${ }^{4}$ and Marco Stampanoni ${ }^{1,3}$ \\ ${ }^{1}$ Swiss Light Source, Paul Scherrer Institut, 5232 Villigen, Switzerland \\ ${ }^{2}$ Centre d'Imagerie BioMédicale, École Polytechnique Fédérale de Lausanne, 1015 Lausanne, Switzerland \\ ${ }^{3}$ Institute for Biomedical Engineering, UZH/ETH Zürich, 8092 Zürich, Switzerland \\ ${ }^{4}$ Laboratory for Micro- and Nanotechnology, Paul Scherrer Institut, 5232 Villigen, Switzerland
}

(Received 10 January 2014; published 11 July 2014)

\begin{abstract}
Multiple scattering represents a challenge for numerous modern tomographic imaging techniques. In this Letter, we derive an appropriate line integral that allows for the tomographic reconstruction of angular resolved scattering distributions, even in the presence of multiple scattering. The line integral is applicable to a wide range of imaging techniques utilizing various kinds of probes. Here, we use $\mathrm{x}$-ray grating interferometry to experimentally validate the framework and to demonstrate additional structural sensitivity, which exemplifies the impact of multiple scattering tomography.
\end{abstract}

PACS numbers: 07.85.Fv, 28.20.Cz, 78.70.Ck, 87.59.-e

In tomographic imaging or scattering methods the occurrence of multiple scatter events is often detrimental to the quality and expressiveness of the obtained results. Multiple scattering affects a wide variety of techniques such as angular-resolved low coherence interferometry [1], Compton scattering tomography [2], optical coherence tomography [3], electron tomography [4], small-angle $\mathrm{x}$-ray or neutron scattering $[5,6]$, and extremely small angle x-ray scatter imaging [7]. At best multiple scattering constitutes an additional source of noise; at worst the assumptions implied by established theoretical frameworks are violated rendering the results physically meaningless. Methods explicitly using multiple scattering like x-ray or neutron dark-field tomography [8-10] do not provide the angular resolved scattering distributions but only the associated widths.

The applicability of tomographic reconstruction requires a line integral that takes the relevant physical processes into account. Until now, a corresponding framework for multiple scattering was not available, which contributes to the issues raised above. In the following, we will address this challenge by deriving an appropriate line integral that solves the infinite number of convolutions associated with multiple scattering.

Scattering can be regarded as a statistical process with the angular deviation $\alpha$ or, equivalently, the momentum transfer $q$ as the random variable and the local (differential) scattering distribution $f(x, y, \alpha)$ as the probability density function. For elastic scattering $\alpha$ and $q$ can be used interchangeably according to $q=\alpha K$ with, $K$, the wave number. In single scattering the direction of the probe is changed only once implying that $\alpha$ constitutes a simple additional parameter for the corresponding line integral. Assuming zero cross-pixel talk and isotropic scattering, i.e., $f(x, y, \theta, \alpha)=f(x, y, \alpha)$, the line integral

$$
\frac{I(t, \theta, q)}{I_{0} T(t, \theta) \Delta \Omega}=\int d s f(s, t, q)
$$

holds true [5,11], which connects the local scattering distribution of the sample $f(s, t, q)$ to the intensity of the projection $I(t, \theta, q)$ normalized by the incident intensity $I_{0}$, the transmission $T(t, \theta)$, and the solid angle $\Delta \Omega$. $(x, y)$ is the frame of reference fixed to the sample while $(s, t)$ parametrizes the beam paths according to the rotation angle $\theta$ (see Fig. 1).

In multiple scattering the scattering angle of the probe at the detector is determined by the sum of the scattering angles of all individual scattering events. Thus, the measurable scattering distribution at the detector $g(t, \alpha, \theta)$ will be given by the convolution of all involved local scattering distributions $f(x, y, \alpha)$ independent of the elasticity of the scattering processes or the monochromaticity of the probes. Thus, after passing $N$ volume elements the resulting

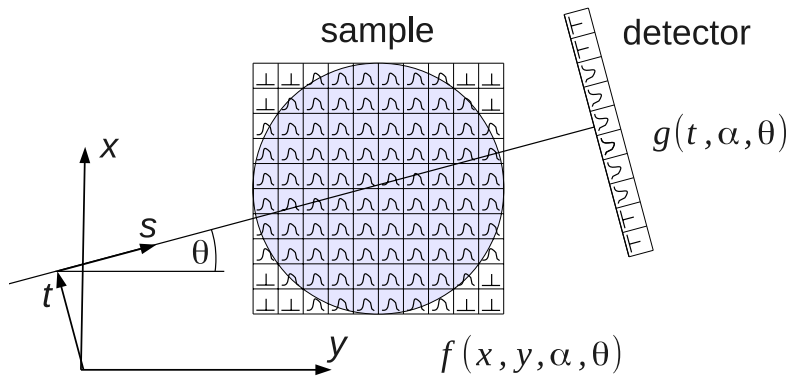

FIG. 1 (color online). Quantities and coordinate systems used in the theoretical derivation of the line integral for multiple scattering. $s$ is the beam direction and the small curves represent the local scattering distributions in the sample $f(x, y, \alpha)$ and at the detector $g(t, \theta, \alpha)$. 
scattering distribution is given by $N$ convolutions with respect to $\alpha$

$$
g(t, \alpha, \theta)=f\left(s_{1}, t, \alpha\right) \Delta s \otimes \ldots \otimes f\left(s_{N}, t, \alpha\right) \Delta s .
$$

Performing the Fourier transform with respect to $\alpha$ leads to

$$
\tilde{g}(t, \theta, \beta)=\prod_{n} \tilde{f}\left(s_{n}, t, \beta\right) \Delta s
$$

with $\beta$ the conjugate variable to $\alpha$. Here, the limit $N \rightarrow \infty$ would imply an infinite product in Fourier space corresponding to an infinite number of convolutions in direct space. In order to calculate them, the auxiliary quantity $\tilde{h}$ with $\tilde{f}\left(s_{n}, t, \beta\right) \Delta s=\exp \left(\tilde{h}\left(s_{n}, t, \beta\right) \Delta s\right)$ is introduced. This is mathematically analogous to the well-known relation between the transmission $T$ and the absorption coefficient $\mu$, i.e., $T \Delta s=\exp (-\mu \Delta s)$. Rewriting Eq. (3)

$$
\tilde{g}(t, \beta, \theta)=\exp \left(\sum_{n} \tilde{h}\left(s_{n}, t, \beta\right) \Delta s\right)
$$

and performing the limit $N \rightarrow \infty$ leads to

$$
\ln \tilde{g}(t, \beta, \theta)=\int d s \tilde{h}(s, t, \beta) .
$$

Equation (5) constitutes a line integral for each $\beta$ and constitutes the basis for multiple scattering tomography. Tomographic reconstruction can be performed by separately reconstructing the real and imaginary part of $\tilde{h}$ for each $\beta$, calculating $\tilde{f}=\exp (\tilde{h})$ and applying the inverse Fourier transform with respect to $\beta$.

The limitations concerning spatial resolution implied by multiple scattering tomography must be discussed separately for resolved and unresolved structures of the sample. The resolved structures (i.e., the voxels in the tomographic slices) correspond to the spatial coordinates $t$ and $s$ in Eq. (5) while $\beta$ constitutes a mere additional parameter for the line integral. Thus, with respect to the resolved structures Eq. (5) represents a standard Radon transform and its limitations regarding tomographic reconstruction are well known as long as the line integral holds true [12]. Therefore, the resolution of the resolved structures is limited by the pixel size of the detector and the number of acquired projection angles $\theta$. Since Eq. (5) is based on the assumption of isotropic scattering, anisotropic scattering will lead to artifacts in the tomographic reconstructions. Occurring anisotropic scattering appears as odd moments in the scattering distributions $g(\alpha)$. Thus, in future studies it may be possible to overcome the disadvantageous influence of anisotropic scattering by artificially setting the odd harmonics of the experimental scattering distributions $g(\alpha)$ to zero prior to tomographic reconstruction.

The unresolved structures of the sample are associated with the scattering angle $\alpha$ and its conjugate $\beta$ in Eq. (5). Since the corresponding structure is unresolved it seems to be more appropriate to refer to structural sensitivity rather than spatial resolution. According to $q=2 \pi / a$, with $a$ the spatial period corresponding to the momentum transfer $q$, and $q=K \alpha$, the largest $\alpha$ available in experiment will determine the structural sensitivity towards unresolved structures in terms of $a$. In practice, the largest available $\alpha$ will be limited by experimental constraints such as acceptance angle or noise, which are determined by the imaging method utilized and experimental details. The case of weak multiple scattering is reflected in our model by scattering distributions $f(x, y, \alpha)$ that are narrow with respect to $\alpha$. These kinds of scattering distributions are adequately reconstructed by Eq. (5) if the available angular resolution of $g(t, \alpha, \theta)$ is sufficient in $\alpha$. Otherwise, the corresponding sample scattering distributions $f(x, y, \alpha)$ will be reconstructed as Dirac's $\delta$ distributions, which can be regarded as an artifact of insufficient angular resolution.

For experimental validation we applied the proposed line integral Eq. (5) to hard x-ray grating interferometry (GI) [13-15]. GI constitutes a recently established phase contrast imaging technique that is especially suitable for imaging soft tissues [16]. Pilot studies show a promising potential for clinical application of GI [17-19]. In principle, the experimental setup consists of two line gratings (Fig. 2).

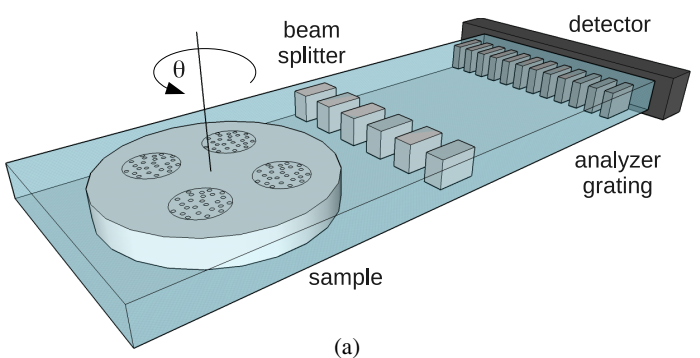

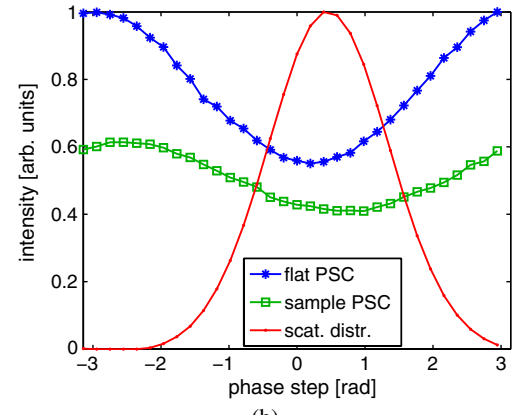

(b)

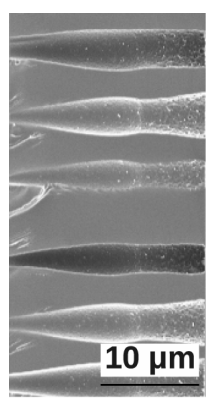

(c)
FIG. 2 (color online). Grating interferometry for USAXS and multiple scattering tomography. (a) Sketch of the experimental setup, which consisted of a sample positioned on a rotation stage, a beam splitter grating, an analyzer grating and a detector (not to scale). (b) Example showing experimental flat and sample PSCs as well as the corresponding normalized scattering distribution retrieved by deconvolution. (c) Scanning electron micrograph of a vertical cut through a structured region of the manufactured sample showing the randomly distributed holes. The x-ray beam direction is perpendicular to the paper plane. 
The beam splitter provides a rectangular-shaped interference pattern via the integer or fractional Talbot effect [20]. A sample present in the beam will introduce a local shift of the interference pattern due to refraction and an analyzer grating is used to scale up this information. By laterally scanning one of the gratings, a so-called phase stepping curve (PSC) is obtained for each detector pixel [cf. Fig. 2(b)]. A Fourier component analysis of the PSCs reveals three complementary images: absorption, differential phase [21], and dark-field contrast [8].

Imaging the angular resolved ultra-small-angle $\mathrm{x}$-ray scattering distribution (USAXS) can be regarded as an extension of the three standard contrasts. For GI the USAXS distributions can be retrieved in two different ways. In Ref. [22] the authors utilized multiple scans with different intergrating distances, model-based data fitting, and consecutive Fourier transform in order to obtain information on unresolved sample features. Later this approach was successfully extended to three dimensions by tomographically reconstructing the individual scans and tracking the reconstructed dark-field values over the different intergrating distances [23]. This approach is based on the assumption of Gaussian-shaped scattering distributions and could be difficult to translate to laboratory setups since a different set of gratings may be required to account for beam divergence.

Recently, we have demonstrated an alternative approach (GI-USAXS) to obtain the angular resolved USAXS distribution within a detector pixel [24] that requires only a single intergrating distance scan and allows for arbitrarily shaped scattering distributions. The corresponding data analysis procedure involves the deconvolution of noisy, periodic data. This challenge has been met by carefully selecting the method and parameters of the deconvolution [25]. It was also demonstrated that GI-USAXS provides a superior contrast to noise ratio for the dark-field contrast, which corresponds to the second moment of the retrieved USAXS distributions, when compared to the standard analysis [26]. The combination of GI-USAXS and multiple scattering tomography preserves the advantageous features of GI-USAXS since Eq. (5) also allows for arbitrarily shaped scattering distributions.

In case of GI-USAXS the scattering distribution related to the sample $f(x, y, \alpha)$ is given by the distribution of refraction angles $\alpha$ contributing to the signal in one detector pixel (i.e., histogram of contributing refraction angles) [24]

$$
f(s, t, \alpha)=\int_{\text {voxel }} d t \delta_{D}[R(t)-\alpha]
$$

with $\delta_{D}$ Dirac's $\delta$ distribution and $R(t)$ the refraction angle as a function of the lateral spatial position $t$. For multiple scattering Eq. (6) requires structurally uncorrelated scattering planes. The dark-field signal at the detector is related to the accumulated scatter width during transmission through the sample; a nonzero dark-field signal within the sample bulk implies the occurrence of multiple scattering. Since this is a typical situation met in experiment, GI constitutes a particularly fitting technique for the investigation of multiple scattering.

The samples consisted of randomly distributed holes in a $19 \mu \mathrm{m}$ thick PMMA layer with known radii and mean distance (cf. Fig. 2) and were manufactured in the Laboratory for Micro- and Nanotechnology (LMN) of the Paul Scherrer Institut (Villigen, Switzerland) by direct write $e$-beam exposure. Within the structured areas of the sample, holes with constant radii were randomly distributed with a given mean distance fulfilling the requirement implied by Eq. (6). Even though the $e$-beam tool (Vistec EBG5000Plus) was operated at $100 \mathrm{kV}$ acceleration voltage, it was not possible to expose and develop holes with required diameter and acceptable shape variation throughout the PMMA layer at one time. The reason for this is the scattering of electrons and the associated energy distribution profile in the volume of the resist. With high contrast developer-IPA:H2O mixture $(7: 3)$ — which was chosen to facilitate steep walls of holes, elongated bulbs of maximum $12 \mu \mathrm{m}$ depth could be obtained. Therefore, an additional overlay exposure and subsequent development step were required. The resulting shape of the hole was inspected by cleaving the control sample and imaging the cross section by a scanning electron microscope, as presented in Fig. 2(c). The theoretical scattering distribution in Fig. 3 was obtained by numerical calculation on the basis of Eq. (6) and the known properties of the sample (i.e., substrate material, hole sizes and distributions) and of the beam (i.e., photon energy).

Tomographic data sets with GI were acquired at the beamline for TOmographic Microscopy and Coherent rAdiology experimenTs (TOMCAT) of the Swiss Light Source of the Paul Scherrer Institut (Villigen, Switzerland) [27]. A photon energy of $25 \mathrm{keV}$ was selected by a double multilayer monochromator. At $25 \mathrm{~m}$ distance from the source, the phase grating with a pitch of $3.98 \mu \mathrm{m}$ was located, which induced a phase shift of $\pi$ into the incident beam. The intergrating distance $(d=121 \mathrm{~mm})$ was close to the optimum in terms of sensitivity to scattering angles [28]. The phase grating as well as the absorption grating (period: $g_{2}=2 \mu \mathrm{m}$ ) were also manufactured in house by the LMN [29]. In order to increase the width of the reconstructed scatter distributions above the $q$ resolution, the raw images were binned in plane three times, which led to an effective pixel size of $52 \mu \mathrm{m}$. The samples were located on a goniometer for horizontal alignment and scanned over 180 deg with 721 projection and 33 phase steps. The resulting flat and sample phase stepping curves were deconvolved in order to retrieve the scattering distributions [cf. Fig. 2(b)] [25] and tomographic reconstruction was performed with a fast regridding technique [30]. 


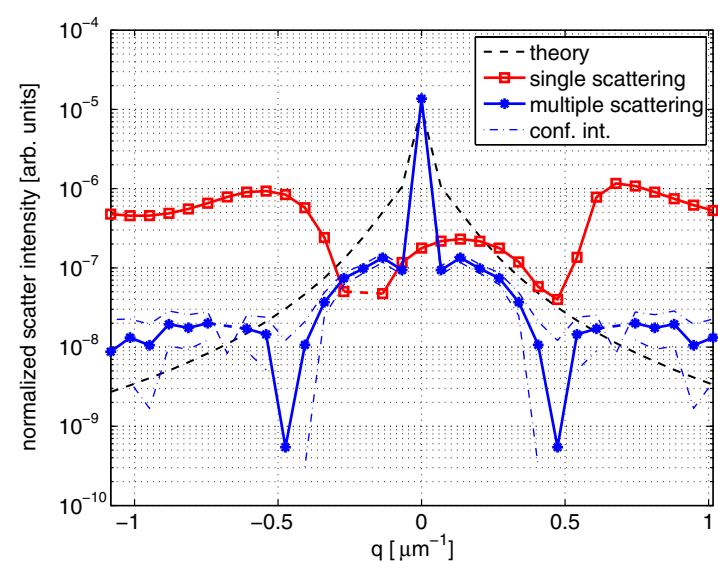

(a)

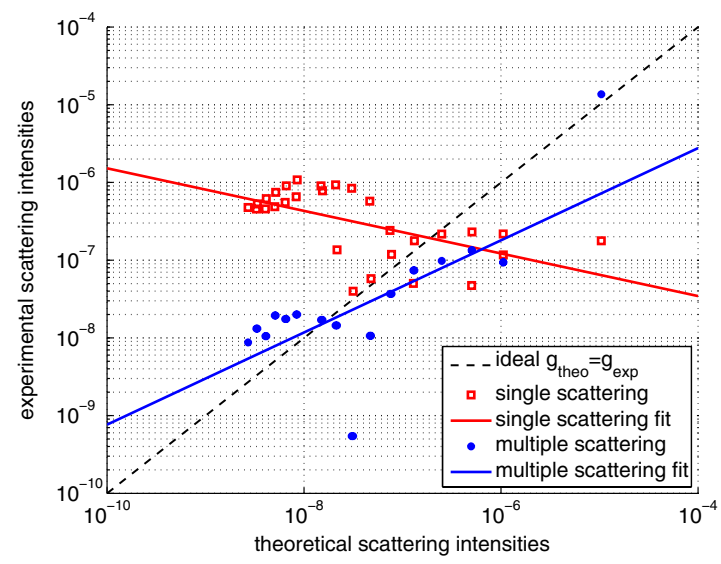

(b)

FIG. 3 (color online). Experimental comparison of single scattering and multiple scattering tomography to the theoretical expectation. (a) Scattering distributions averaged over one structured area of the sample assuming single [Eq. (1)] or multiple [Eq. (5)] scattering and of theoretical expectations from the known properties of the sample and Eq. (6). For the case of multiple scattering the confidence interval is indicated by a dashdotted line. Distributions were normalized to $\int f(q) d q=1$. Negative values are due to noise and are not shown but indicated by dashed lines. (b) Scatter plot comparing the scattering intensities using the single as well as the multiple scattering model to the theoretical expectation.

Figure 3(a) compares the scattering distributions averaged over one structured area of the sample [similar to rectangles in Fig. 4(a)] as retrieved by single scattering [Eq. (1)] and multiple scattering [Eq. (1)] to the theoretical expectation according to Eq. (5). The confidence interval for the case of multiple scattering shows the reliability of the retrieved data and was determined by the standard deviation within the region of interest. While a notable deviation of the experimental results from the theoretical expectation is observed for the proposed line integral, it is obvious that the analysis based on multiple scattering shows superior predictive power over the single scattering approach. This superiority of multiple scattering tomography is also reflected by the scatter plot in Fig. 3(b). Linear

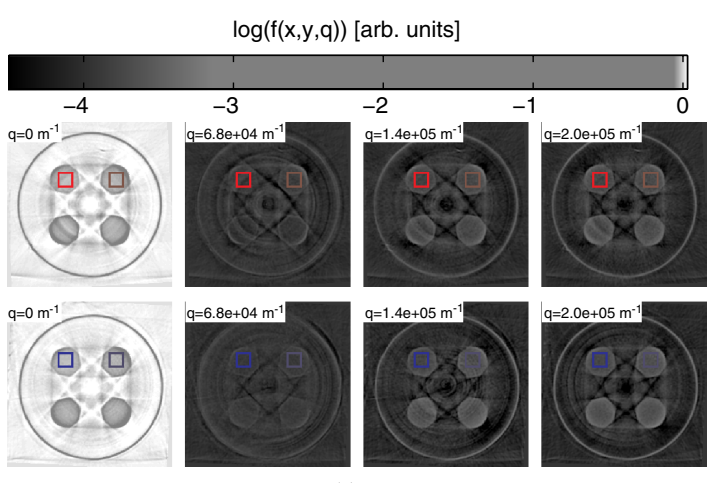

(a)

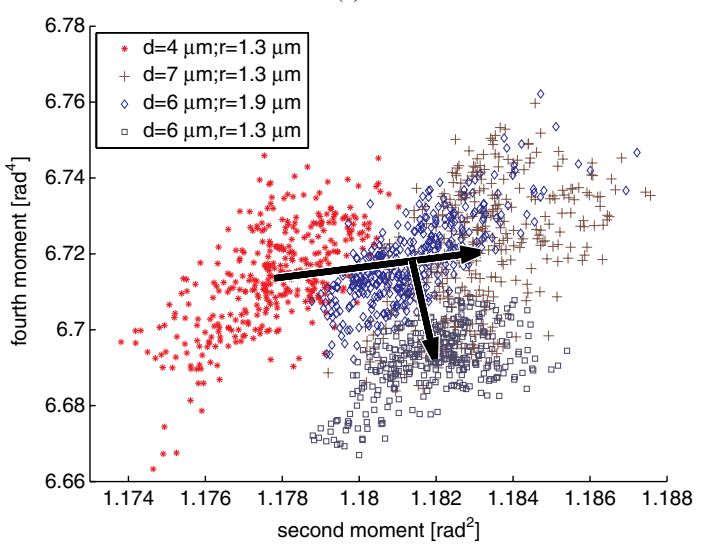

(b)

FIG. 4 (color online). Tomographic reconstruction of local scatter distributions of samples showing multiple scattering. (a) Tomographic slices of reconstructed scatter distributions for two PMMA samples each containing four areas of unresolved holes with either varying mean distance (top) or varying mean radius (bottom). Each image shows the scatter contrast for a specific $q$ value. (b) Scatter plot of the second and fourth moment of the local scattering distributions within the areas indicated by the squares in (a). $d$ denotes the mean distance and $r$ the mean radius of the randomly distributed holes for the four structured areas. A priori information about the second sample was used to horizontally adjust the blue colored data sets as to meet the expected relative location. Arrows connect the centers of mass of the data sets showing the influence of varying mean distance (horizontally) and mean radius (vertically) of hole distribution.

fits confirmed a much better agreement between theory and experiment for the multiple scattering approach (slope $=0.6 \pm 0.1$ ) than for the single scattering approach (slope $=-0.3 \pm 0.1$ ).

In order to validate our framework we utilized the Kolmogorov-Smirnov test [31], which determines if two data sets are likely to originate from the same underlying distribution. It uses the largest difference of the cumulative probability density distributions as a test statistic. The test can be used to compare theory to experiment by identifying the theoretical expectations with one data set and experimental results with the other. Here, the results of multiple scattering were compatible with the theoretical expectations on a 0.05 significance level, while single scattering 
was rejected. These results indicate the validity of the proposed line integral.

We attribute the remaining bias of the multiple scattering approach to the specifics of its application to GI. Due to the binary shape of the utilized gratings, the even harmonics of the PSCs are zero and the corresponding information is missing in experimental scattering distributions $g(t, \alpha, \theta)$. Naturally, this will negatively affect the quantitative results of tomographic reconstruction. However, this limitation can be overcome by utilizing nonbinary gratings [32], which will recover the even harmonics of the PSCs and increase the agreement between theoretical predictions and experimental results.

As examples for the impact of the line integral for multiple scattering we like to point out two resulting advancements for grating interferometry. First, each point of the scattering distributions in Fig. 3(a) corresponds to a tomographic slice. Thus, the number of complementary tomographic contrasts was increased from previously 3 to 16 here and potentially hundreds. Second, we will demonstrate the usefulness of the increased information content by revealing additional structural information of subpixel features in the following.

We manufactured two samples: one where the mean distance of the holes was varied over the structured areas while the mean radii were kept constant (indicated by red markers in Fig. 4) and another sample with the opposite behavior (blue markers in Fig. 4). The visible streaklike artifacts, which connect the edges of the structured areas, are due to the violation of the isotropic scattering assumption in Eq. (5). For a specific point of an edge refraction mainly occurs at projection angle $\theta$, where the edge is perpendicular to the detector line. Thus, refraction constitutes anisotropic scattering in the present context and the effect is largest in the projections, where two edges coincide. Therefore, refraction appears as streaklike artifacts in the reconstructed slices. As mentioned above this may be overcome by artificially setting the odd moments of the scattering distributions to zero prior to tomographic reconstruction.

The second moment of the local scattering distributions is plotted against the fourth moment as a scatter plot in Fig. 4(b). While changing the mean radius of the holes corresponds to a vertical movement in the scatter plot, changing the mean distance corresponds to a horizontal movement. The effects of hole radii and mean distance can be separated. Therefore, we have demonstrated that multiple scattering tomography applied to GI-USAXS provides structural sensitivity beyond what is accessible by the standard scan and analysis procedures in GI and without the need for multiple scans with different intergrating distances. Future investigations will reveal if this qualitative result can be improved to provide quantitative measurements.

In conclusion, we derived a line integral for multiple scattering tomography that is based on the convolution of local scattering distributions. The proposed line integral is based on three assumptions: isotropic scattering, mutually incoherent scatter events, and negligible cross-pixel talk. However, the framework is independent of the exact type of probe (x rays, neutrons, optical photons, etc.) and only requires the availability of the pixelwise angular resolved scattering distribution. This requirement is fulfilled by small angle x-ray [33] and neutron [6] scattering, grating-based [8] and crystal-based [9] imaging methods and, thus, the framework is directly applicable to these techniques. Therefore, we expect a widespread application of the proposed line integral as well as a strong impact on current research that is aimed at solving the challenge of multiple scattering.

We would like to acknowledge Christian Grünzweig, Oliver Bunk, Andreas Menzel, and Zhentian Wang (all of Paul Scherrer Institut, Villigen, Switzerland) for insightful discussions. This study was supported by Centre d'Imagerie BioMédicale (CIBM) of the UNIL, UNIGE, HUG, CHUV, and EPFL and the Leenaards and Jeantet Foundations. This work has been partially supported by ERC Grant No. ERC-2012-StG 310005-PhaseX.

* peter.modregger@psi.ch

[1] A. Wax, C. Yang, V. Backman, M. Kalashnikov, R. R. Dasari, and M. S. Feld, J. Opt. Soc. Am. A 19, 737 (2002).

[2] S. Norton, J. Appl. Phys. 76, 2007 (1994).

[3] D. Huang et al., Science 254, 1178 (1991).

[4] R. McIntosh, D. Nicastro, and D. Mastronarde, Trends Cell Biol. 15, 43 (2005).

[5] T. H. Jensen, M. Bech, O. Bunk, M. Thomsen, A. Menzel, A. Bouchet, G. Le Duc, R. Feidenhansl, and F. Pfeiffer, Phys. Med. Biol. 56, 1717 (2011).

[6] M. Strobl, W. Treime, and A. Hilger, Appl. Phys. Lett. 85, 488 (2004).

[7] A. Olivo, F. Arfelli, D. Dreossi, R. Longo, R. H. Menk, S. Pani, P. Poropat, L. Rigon, F. Zanconati, and E. Castelli, Phys. Med. Biol. 47, 469 (2002).

[8] F. Pfeiffer, M. Bech, O. Bunk, P. Kraft, E. F. Eikenberry, Ch. Brönnimann, C. Grünzweig, and C. David, Nat. Mater. 7, 134 (2008).

[9] H. Suhonen, M. Fernández, A. Bravin, J. Keyriläinen, and P. Suortti, J. Synchrotron Radiat. 14, 512 (2007).

[10] M. Strobl, C. Grünzweig, A. Hilger, I. Manke, N. Kardjilov, C. David, and F. Pfeiffer, Phys. Rev. Lett. 101, 123902 (2008).

[11] C. G. Schroer, M. Kuhlmann, S. V. Roth, R. Gehrke, N. Stribeck, A. Almendarez-Camarillo, and B. Lengeler, Appl. Phys. Lett. 88, 164102 (2006).

[12] A.C. Kak and M. Slaney, Principles of Computerized Tomographic Imaging (IEEE Press, New York, 1988).

[13] C. David, B. Nöhammer, H. H. Solak, and E. Ziegler, Appl. Phys. Lett. 81, 3287 (2002).

[14] A. Momose, S. Kawamoto, I. Koyama, Y. Hamaishi, K. Takai, and Y. Suzuki, Jpn. J. Appl. Phys. 42, L866 (2003). 
[15] F. Pfeiffer, T. Weitkamp, O. Bunk, and C. David, Nat. Phys. 2, 258 (2006).

[16] F. Pfeiffer, O. Bunk, C. David, M. Bech, G. Le Duc, A. Bravin, and P. Cloetens, Phys. Med. Biol. 52, 6923 (2007).

[17] T. Donath, F. Pfeiffer, O. Bunk, C. Grünzweig, E. Hempel, S. Popescu, P. Vock, and C. David, Invest. Radiol. 45, 445 (2010).

[18] M. Stampanoni, Z. Wang, T. Thüring, C. David, E. Roessl, M. Trippel, R. A. Kubik-Huch, G. Singer, M. K. Hohl, and N. Hauser, Invest. Radiol. 46, 801 (2011).

[19] T. Michel et al., Phys. Med. Biol. 58, 2713 (2013).

[20] P. Modregger, B. R. Pinzer, Z. Wang, and M. Stampanoni, in Emerging Imaging Technologies In Medicine. edited by M. A. Anastasio and P. La Riviere (CRC Press, Boca Raton, 2013).

[21] T. Weitkamp, A. Diaz, C. David, F. Pfeiffer, M. Stampanoni, P. Cloetens, and E. Ziegler, Opt. Express 13, 6296 (2005).

[22] W. Yashiro, Y. Terui, K. Kawabata, and A. Momose, Opt. Express 18, 16890 (2010).

[23] W. Yashiro, S. Harasse, H. Kuwabara, K. Kawabata, and A. Momose, AIP Conf. Proc. 1466, 211 (2012).
[24] P. Modregger, F. Scattarella, B. R. Pinzer, C. David, R. Bellotti, and M. Stampanoni, Phys. Rev. Lett. 108, 048101 (2012).

[25] F. Scattarella, S. Tangaro, P. Modregger, M. Stampanoni, L. De Caro, and R. Bellotti, Physica Medica 29, 478 (2013).

[26] T. Weber, G. Pelzer, F. Bayer, F. Horn, J. Rieger, A. Ritter, A. Zang, J. Durst, G. Anton, and T. Michel, Opt. Express 21, 18011 (2013).

[27] S. A. McDonald, F. Marone, C. Hintermüller, G. Mikuljan, C. David, F. Pfeiffer, and M. Stampanoni, J. Synchrotron Radiat. 16, 562 (2009).

[28] P. Modregger, B. R. Pinzer, T. Thüring, S. Rutishauser, C. David, and M. Stampanoni, Opt. Express 19, 18324 (2011).

[29] C. David, J. Bruder, T. Rohbeck, C. Grun̈zweig, C. Kottler, A. Diaz, O. Bunk, and F. Pfeiffer, Microelectron. Eng. 84, 1172 (2007).

[30] F. Marone and M. Stampanoni, J. Synchrotron Radiat. 19, 1029 (2012).

[31] F. J. Massey, J. Am. Stat. Assoc. 46, 68 (1951).

[32] A. Yaroshenko et al., Opt. Express 22, 547 (2014).

[33] O. Bunk, M. Bech, T. H. Jensen, R. Feidenhansl, T. Binderup, A. Menzel, and F. Pfeiffer, New J. Phys. 11, 123016 (2009). 